\title{
Human pressure on rivers is increasing worldwide and threatens water security
}

\author{
SERENA CEOLA ${ }^{1}$, FRANCESCO LAIO ${ }^{\mathbf{2}}$ \& ALBERTO MONTANARI ${ }^{\mathbf{1}}$ \\ 1 Department DICAM, University of Bologna, via del Risorgimento 2, I-40136 Bologna, Italy \\ serena.ceola@unibo.it \\ 2 Dipartimento di Ingegneria dell'Ambiente, del Territorio e delle Infrastrutture, Politecnico di Torino, \\ C.so Duca degli Abruzzi, 24, I-10129, Torino, Italy
}

Water security problems are becoming more and more challenging worldwide for several reasons, including population growth, urbanization, land-use change, unsustainable water use and climate change. All the above factors imply an increase in the human exploitation of water resources and an increasing anthropogenic impact on rivers, flood plains, and fresh groundwater. In fact, a striking problem in many countries is the result of destructive changes to the physical structure of the riverine environment. Most important rivers all over the world have been regulated, dammed and diverted. Moreover, the intensive urbanization of flood plains implies the need to protect human settlements against floods, which is often achieved through river training. All these anthropogenic effects are referred to as "human pressure on rivers", which has a deleterious effect on water security. In fact, human-induced alterations to rivers can have serious consequences for freshwater quality and quantity by altering the river flow regime and riverine ecosystem. Therefore, the assessment of the human pressure on rivers is extremely important to locate areas where water resources are threatened and subjected to rapidly increasing anthropogenic effects. However, studies are typically carried out at local or regional scales which makes it difficult to consistently identify worldwide patterns.

We propose a novel approach to explore the human pressure on rivers worldwide at a fine spatial resolution by using night-light data. Night lights have been employed in several studies as a proxy for population density, economic development, electric power consumption, poverty and development status, urban extent, and for other environmental analyses, such as the assessment of light pollution. Herein, we associate night-light data and digital information about the geographical coordinates of the river network to locate the areas where the human pressure on rivers is currently increasing, therefore raising concerns for water security.

In detail, we map global night lights in the immediate proximity of the river network from 1992 to 2012, with a spatial resolution of $1 \mathrm{~km}^{2}$. Then, we infer temporal trends in night lights all over the world and in selected regions, by determining the temporal evolution of spatiallyaveraged light intensities for each region of interest.

On a global scale we find an overall positive trend of river network night lights, corresponding to a $1.2 \%$ increase per year which undoubtedly points out a worldwide rise in water resources exploitation. In order to identify possible spatial patterns, we analyse the temporal tendencies at both the continental and national scales. For the sake of comparison, we normalize each annual value (spatially averaged over the region of interest) dividing it by the corresponding multi-year average. Trend coefficients for normalized night lights represent percentage-per-year variations in light intensity.

Interestingly, a clear differentiation emerges: whether considering country-based data or continental-scale values, Africa, Asia and South America reveal appreciable positive trends, Oceania and Europe exhibit a moderately positive trend, whereas North America presents a slightly negative tendency. These outcomes likely resemble a distinction based on the economic development of single continents, where considerably positive night-light trends coincide with developing and emerging regions, characterized by an overall lower-middle income, whereas slightly positive and slightly negative night-light trends mainly correspond to developed continents, with an overall middle-high income. Decreasing night-light trends may be also induced 
by light pollution reduction strategies such as the policy-driven initiatives promoted in the UK and in several USA states.

The temporal enhancement of night lights close to rivers during the last 20 years suggests a potential increment of challenges for water security. Regions in the African continent, where riverine night lights are markedly increasing in time, provide interesting examples. The Nile River and Delta region represent, for instance, a peculiar hot spot characterized by a significant increment in night lights and therefore in water exploitation.

Our study shows that satellite-based night-light maps may offer a strong propellant for the analysis of human pressure on rivers. Indeed, the results clearly reveal a consistent characterization of enhanced anthropogenic pressure close to streams and rivers, as derived from night-light data.

We believe that our analysis may provide a benchmark for the identification of regions justifying priority attention. 